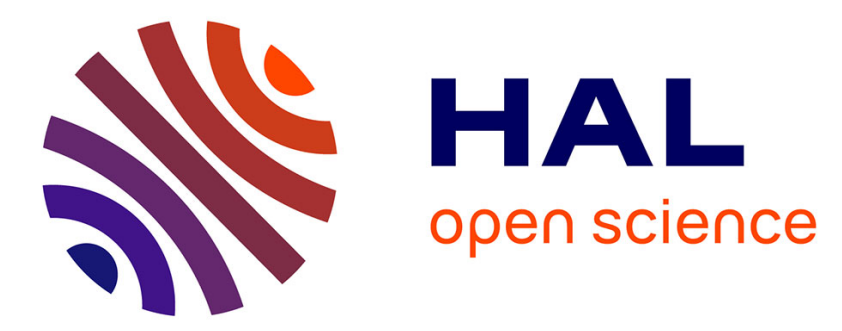

\title{
Waveguide for Lamb Waves Based on Whispering-Gallery Mode
}

Weitao Yuan, Jinfeng Zhao, Bernard Bonello, Yongdong P Pan, Zheng Zhong

\section{To cite this version:}

Weitao Yuan, Jinfeng Zhao, Bernard Bonello, Yongdong P Pan, Zheng Zhong. Waveguide for Lamb Waves Based on Whispering-Gallery Mode. 2018 IEEE International Ultrasonics Symposium (IUS), Oct 2018, Kobe, Japan. 10.1109/ULTSYM.2018.8579950 . hal-02297918

\section{HAL Id: hal-02297918 https://hal.sorbonne-universite.fr/hal-02297918}

Submitted on 26 Sep 2019

HAL is a multi-disciplinary open access archive for the deposit and dissemination of scientific research documents, whether they are published or not. The documents may come from teaching and research institutions in France or abroad, or from public or private research centers.
L'archive ouverte pluridisciplinaire HAL, est destinée au dépôt et à la diffusion de documents scientifiques de niveau recherche, publiés ou non, émanant des établissements d'enseignement et de recherche français ou étrangers, des laboratoires publics ou privés. 


\title{
Waveguide for Lamb waves based on whispering-gallery mode
}

\author{
Weitao Yuan ${ }^{1}$, Jinfeng Zhao ${ }^{{ }^{*}}$, Bernard Bonello ${ }^{2}$, Yongdong Pan ${ }^{1}$, Zheng Zhong ${ }^{1}$ \\ ${ }^{I}$ School of Aerospace Engineering and Applied Mechanics, Tongji University \\ 100 Zhangwu Road, 200092 Shanghai People's Republic of China \\ ${ }^{2}$ Sorbonne Université, UPMC Université Paris 06 (INSP-UMR CNRS 7588), \\ 4 Place Jussieu 75005 Paris, France \\ E-mail: jinfeng.zhao@tongji.edu.cn
}

\begin{abstract}
We proposed three kinds of waveguides namely straight waveguide, Z-shaped waveguide and Pi-shaped waveguide in an otherwise perfect phononic crystal. We analyzed numerically the transmissivity and the displacement field distribution behind the waveguides. We demonstrated that these three waveguides are efficient for guiding the lowest-order antisymmetric Lamb mode $\left(\mathrm{A}_{0}\right)$, based on the whispering-gallery modes in the hollow pillars that comprise the route line. Our approach provides a simple method to design sophisticated waveguides.
\end{abstract}

\section{Introduction}

As a result of the tunable dispersion relation, phononic crystals (PCs) are widely used to manipulate the propagation of elastic/acoustic waves, and many achievements such as acoustic lens [1, 2], diodes [3], and waveguides [4] have been reported. In general, embedding defects into the otherwise perfect PCs can lead to the defect modes which are very attractive, because when falling in the stop band they retain the ability of allowing waves to pass the route line, suitable for wave guiding and filtering. Such a success has been witnessed by many achievements through the creation of an accurately designed linear or L-shaped defect [5-8], for different waves such as bulk waves [9], Lamb waves [10-12] and surface acoustic waves $[13,7]$. However, this kind of waveguides feature a broad pass band and therefore a low quality factor.

Another approach to construct waveguides is using resonators as defects [14-18]. Yuan et al [17] show the experimental evidence that replacing one row of solid pillars with hollow pillars in an otherwise perfect PCs gave rise to high quality factor waveguides owing to the 
whispering-gallery modes (WGMs) in the hollow pillars. In the acoustic filed, although WGMs feature the manifold polarization symmetries, including the quadrupolar, hexapolar, and octopolar symmetries, only one type of quadrupolar WGMs is generated.

In this work, we investigate the straight waveguide, Z-shaped waveguide and Pi-shaped waveguide consisting of hollow pillars erected along accurately designed route lines on a PC plate for $\mathrm{A}_{0}$ mode Lamb wave. Based on the generation of the quadrupolar WGMs in the hollow pillars, we study the performance of these waveguides.

\section{Band structures}

(a)

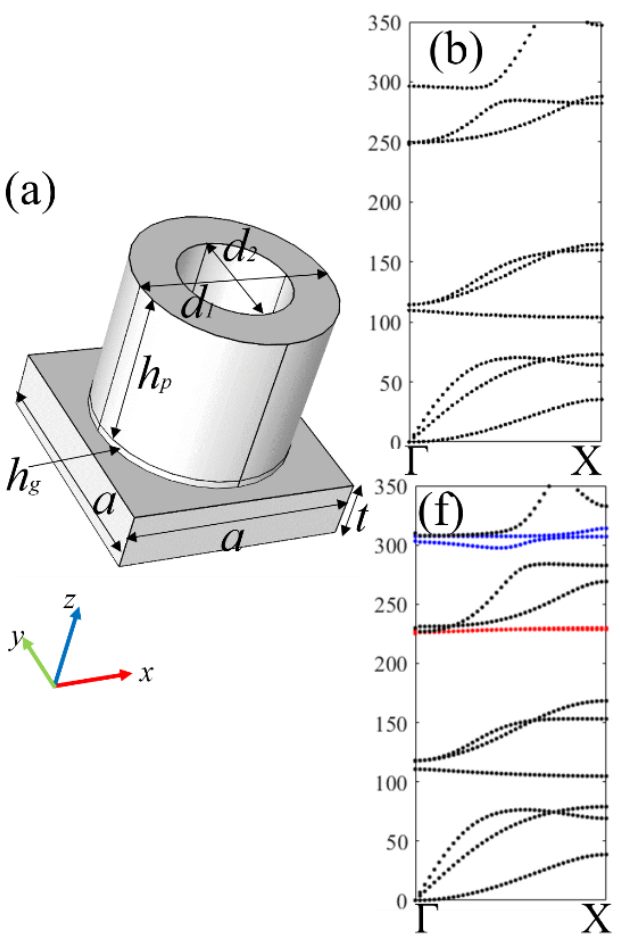

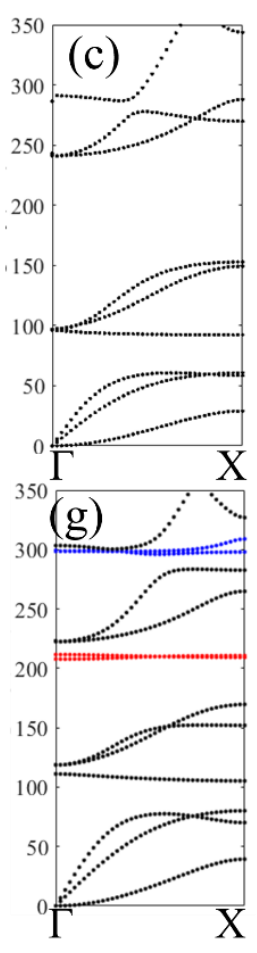

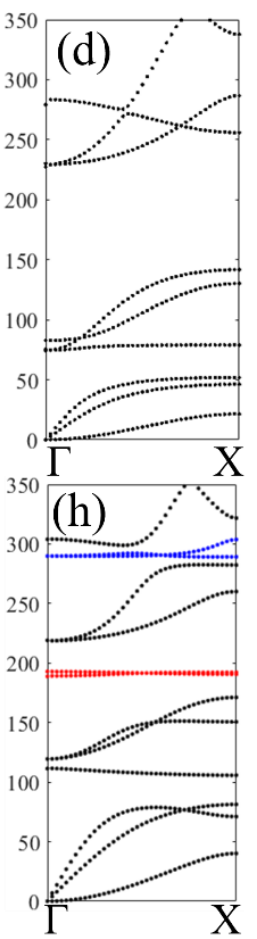

Figure 1 (a) Unit cell of the square-latticed hollow PC plate. (b) - (i) Band structures of the PCs along $\Gamma \mathrm{X}$ direction wherein $a=6, t=1.5, h_{g}=0.135, d_{1}=5$. (b) - (e) Solid pillars $\left(d_{2}=0\right)$ with height $h_{p}=$ 2.4, $h_{p}=3, h_{p}=4, h_{p}=5$, respectively. (f) - (i) Hollow pillars with height $h_{p}=2.4$ and inner diameters $d_{2}=2.4,2.6,2.8$ and 3 respectively. All dimensions are in unit $\mathrm{mm}$.

Firstly, we investigated the evolution of the stop band of PCs plate with solid or hollow pillars in order to optimize the design. Considering we used epoxy adhesive to cement solid or hollow steel pillars on a thin aluminum slab in square lattice, the PCs are named as hollow or solid PC according to whether it includes hollow or solid pillars in the unit. Fig. 1(a) shows the unit 
cell of the square-latticed hollow PC plate whose geometrical parameters are the lattice constant $a$, the plate thickness $t$, the height of the lower epoxy resin pillar $h_{g}$ and height of the upper steel pillar $h_{p}$. We assume the resin layer and steel pillars have the same outer diameter $d_{l}$ and same inner diameter $d_{2}$. We set (in mm) $a=6, t=1.5, h_{g}=0.135$ [19], and $d_{l}=5$ throughout this work. The physical parameters of steel, aluminum and epoxy resin are Young's modulus (in GPa) 226, 67.7, 5.1, Poisson's ratio 0.29, 0.35, 0.38, and the density (in $\left.\mathrm{g} \square \mathrm{cm}^{-3}\right) 7.75,2.7,1.2$, respectively.

All the dispersion curves are computed using the finite element code COMSOL Multiphysics ${ }^{\circledR}$. Figs. 1(b) - 1(e) show the evolution of the band structure along $\Gamma \mathrm{X}$ of Brillouin zone (BZ) against the height $h_{p}$ of solid steel pillars, and it comes to the result that the higher the pillar is the lower the dispersion curves are. Then we calculated the dispersion curves of hollow PC plate with height $h_{p}=2.4 \mathrm{~mm}$ when the inner diameters $d_{2}=2.4,2.6,2.8$, $3.0 \mathrm{~mm}$, respectively. In comparison to the solid PC in Fig. 1(b), the hollow PC in Fig. 1(f) have four more branches marked by red and blue colors. These additional branches, as shown in Figs. 1(f) - 1(i), are shifted to the lower frequencies as the inner diameter $d_{2}$ increases. From Fig. 1(h), we can find out that when $h_{p}=2.4 \mathrm{~mm}$ and $d_{2}=2.8 \mathrm{~mm}$, two flat branches marked by red color appear in hollow PC around $190 \mathrm{kHz}$ at almost the center of the stop band, opening between $164 \mathrm{kHz}$ and $249 \mathrm{kHz}$, of its solid PC counterpart in Fig. 1(b). For feasibility and simplicity, we thus choose the hollow pillars with height $h_{p}=2.4 \mathrm{~mm}$ and inner diameter $d_{2}=2.8 \mathrm{~mm}$ throughout this work.

(a)

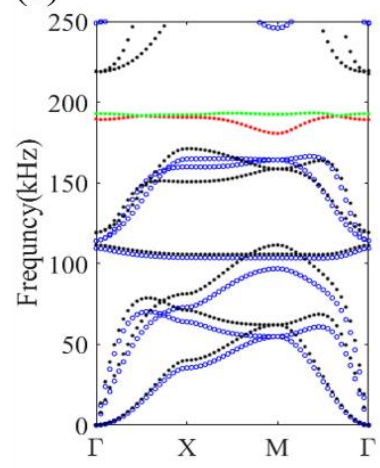

(b)

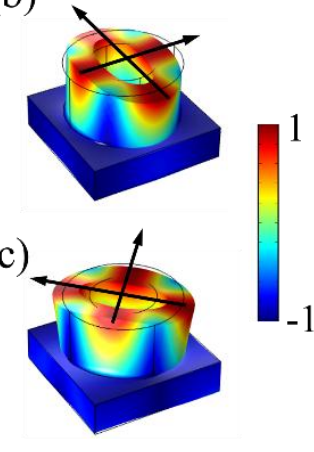

Figure 2 (a) Band structures of PCs plate of solid (blue circle lines, $d_{2}=0$ ) or hollow pillars (dotted lines, $d_{2}=2.8 \mathrm{~mm}$ ) with $a=6, t=1.5, h_{g}=0.135, d_{l}=5, h_{p}=2.4 \mathrm{in} \mathrm{mm}$. Normalized distribution of total displacement $u_{t}$ and deformation at point X of the BZ for (b) WGM 1 and (c) WGM 2. 
The blue circle lines in Fig. 2(a) indicate the band curves of solid PC plate while the dotted lines are for the band structure of hollow PC plate. In comparison to the case for the solid PC, two flat branches appear in between 181 and $192.9 \mathrm{kHz}$ in Fig. 2(a), because of the occurrence of quadrupolar WGMs in the hollow pillars. Figs. 2(b) and 2(c) show the normalized distribution of total displacement field $u_{t}$ and the deformation of the unit cell at the $\mathrm{X}$ point of the BZ, both featuring quadrupolar WGMs. These two modes are named as WGM 1 and WGM 2, respectively, in this work. As shown by the black arrows in Fig. 2(b), the WGM 1 has two polar axes parallel to the $x$ - and $y$ axes while the WGM 2 in Fig. 2(c) has two polar axes featuring $45^{\circ}$ to the $x$ or $y$ axis. From Fig. 2(b) and Fig. 2(c), it is clear that the energy is strongly localized in hollow pillars and decreases from the top surface to the foot of hollow pillar.

\section{IIISamples and Numerical Results}

(a)

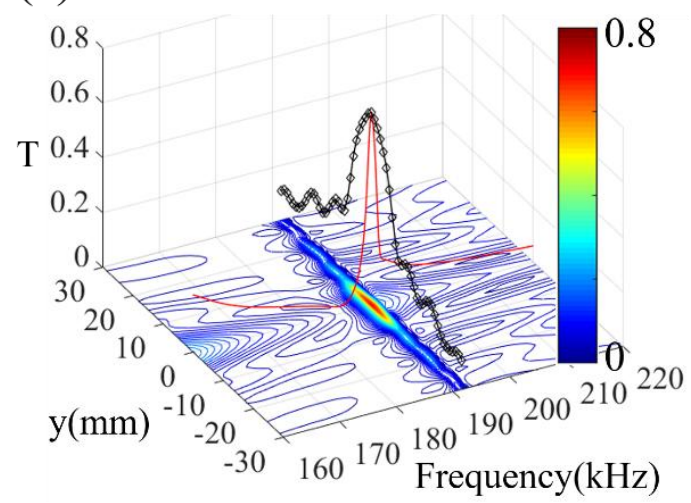

(b)

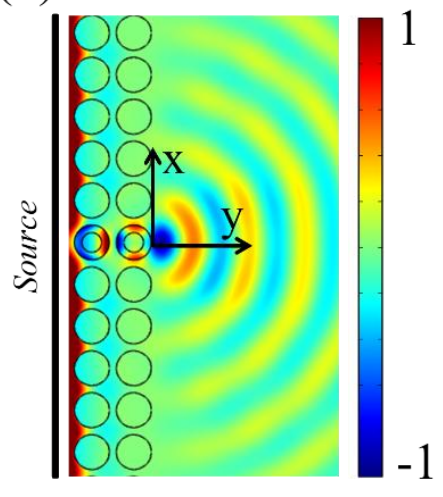

Figure 3 (a) Transmission spectrum computed at $x=6 \mathrm{~mm}$ behind straight waveguide. The red line is the transmissivity at $x=6 \mathrm{~mm}$ and $y=0$. The diamond lines correspond to the profile at the peak in the transmitted spots. (b) Normalized distribution of out-of-plane displacement $u_{z}$ at $f=191.5 \mathrm{kHz}$ where the transmissivity is maximum.

We firstly built a straight waveguide consisting straightly aligned hollow pillars. We simulated the wave propagation through the waveguide with a finite element method. To mimic $\mathrm{A}_{0}$ mode source, we set a transverse line (11a long) at $7.5 \mathrm{~mm}$ before the inlet interface, upon which we applied a force polarized in the $z$ axis with a Gaussian profile along $y$ axis. We then made the calculation over the frequency range $160-220 \mathrm{kHz}$. We defined the 
transmissivity as $T=U / U_{0}$ where $U_{0}$ stands for the amplitude of the source signals on a pure aluminum substrate at a distance off the source line the same as the pillars and $U$ corresponds to the amplitude of the signals at the outlet of the waveguides.

Fig. 3(a) shows the computed transmission spectrum as a function of the position along the $y$ axis at $x=6 \mathrm{~mm}$ behind the waveguide. The red line is the transmissivity at $x=6 \mathrm{~mm}$ and $y=$ 0 . It indicates firstly that the structure has an almost total extinction of the signal except the spot region in the band gap of the PC made of solid pillars. Secondly, a spot emerges at frequencies $f=191.5 \mathrm{kHz}$ on the central axis at $y=0$ in range $189-200 \mathrm{kHz}$ with maximum transmissivity $T_{\max }=0.70$ as diamond line (symmetric against $y$ axis) in Fig. 3(a), matching well the WGM branches in Fig. 2(a).

Fig. 3(b) shows the distribution of out-of-plane displacement $u_{z}$ normalized to the amplitude of incident wave at frequency $f=191.5 \mathrm{kHz}$. It demonstrates the wave propagation in the route line consisting of hollow pillars and the generation of quadrupolar WGM 1 in hollow pillars. In this case, the wave guiding by the WGM 1 is clearly evidenced. Because of the symmetric conditions, the WGM 2 whose polar directions are at an angle of $45^{\circ}$ to the $x$ - and $y$ axes (Fig. 2(c)) cannot be observed in this simulation [17]. Also, the spot can be well understood since the underlying mechanism, i.e., upon the excitation of the quadrupolar WGM 1, the hollow pillar shall reemit a quadrupole $\mathrm{A}_{0}$ mode along both the $x$ and $y$ axes in the rear of the waveguide as shown in Fig. 3(b).

(a)

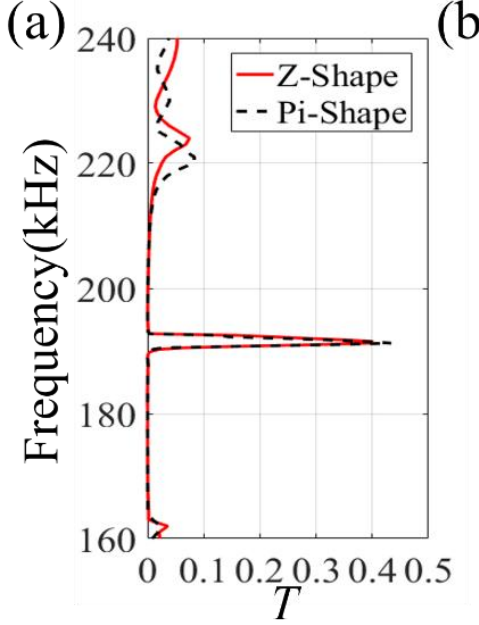

(b)

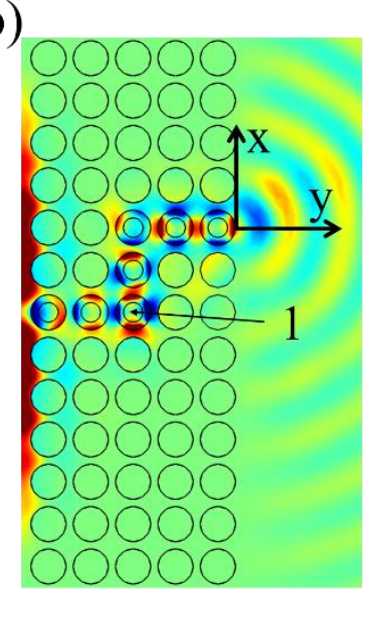

(c)

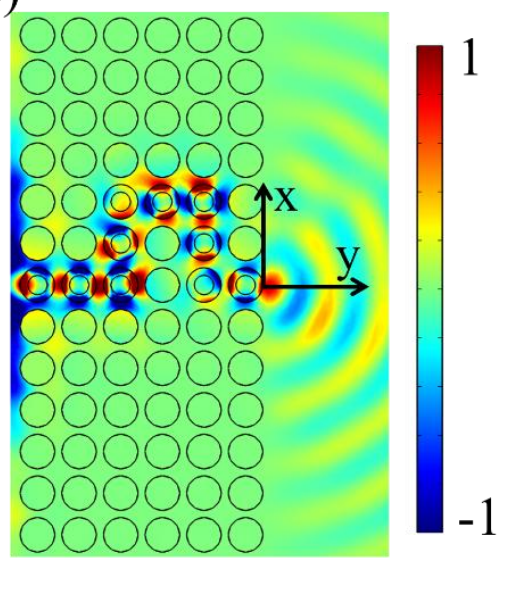

Figure 4 (a) Transmission spectrum computed at $x=6 \mathrm{~mm}$ behind the Z-shaped waveguide (red solid 
line) and Pi-shaped waveguide (black dashed line). Normalized distribution of $u_{z}$ at $f=191.5 \mathrm{kHz}$ for both Z-shaped waveguide (b) and Pi-shaped waveguide (c).

We then built up Z-shaped and Pi-shaped waveguides as shown in Figs. 4(b) and 4(c), respectively. The red solid line and black dashed line in Fig. 4(a) stands for the computed transmissivity at $x=6 \mathrm{~mm}$ behind the hollow pillars at the outlet of Z-shaped and Pi-shaped waveguides, respectively. In both cases, the transmission peak appears at frequency $f=191.5$ $\mathrm{kHz}$, featuring the same frequency as that in straight waveguide, with however a little smaller maximum transmissivity $T_{\max }=0.40$ (red solid line) and $T_{\max }=0.44$ (black dashed line). The frequency of these peak matches well the WGM branches in Fig. 2(a).

Figs. 4(b) and 4(c) show the normalized distribution of $u_{z}$ at frequency $f=191.5 \mathrm{kHz}$ for Z-shaped and Pi-shaped waveguides, respectively. Both figures demonstrated the wave propagation along the designed route line consisting of hollow pillars and the generation of WGMs. At the rear of these two waveguides, the displacement field $u_{z}$ is asymmetric against the $y$ axis, which is different from the case for straight waveguide. This can be understood by analyzing the WGMs in hollow pillars. For example, in Fig. 4(b) the constraint from the solid pillars is asymmetric against $y$ axis or either $x$ axis, and in return the WGMs generated in hollow pillar (labeled as 1) at corner are not only WGM 1 or WGM 2, but a combination of these two. Then the hollow pillar reemits a quadrupole $\mathrm{A}_{0}$ mode featuring an angle to the $x$ and $y$ axes. Due to the symmetry conditions, the polarization axes of the generated WGMs in hollow pillars behind hollow pillar 1 also featuring an angle to $x$ - and $y$ axes. In return, the reemitted $\mathrm{A}_{0}$ mode and the displacement field $u_{z}$ at the rear of the waveguide is asymmetric against the $y$ axis. After all, these two kinds of waveguides are shown to be efficient for wave guiding.

\section{IVCONCLUSIONS}

In summary, we proposed three kinds of whispering-gallery mode based waveguides by inserting an accurately designed route line consisting of hollow pillars in between a 
square-latticed PC plate. We then demonstrated numerically the efficiency of straight, Z-shaped and Pi-shaped waveguides for guiding the lowest-order antisymmetric Lamb mode. Our results suggest that WGMs could be used in waveguides featuring sophisticated and configurable shapes and open a simple way to design them. These waveguides can be used in integrated devices such as telecommunications, sensors and medical imaging.

\section{Acknowledgements}

Financial support from the Natural Science Foundation of China (Grant No. NFSC 11602174) and the Fundamental Research Funds for the Central Universities (Grant No. 2016KJ008) is gratefully acknowleged.

\section{Reference}

[1] Z. He, F. Cai, Y. Ding and Z. Liu, "Subwavelength imaging of acoustic waves by a canalization mechanism in a two-dimensional phononic crystal," Appl. Phys. Lett., vol. 93, (23), pp. 233503, 2008. [2] J. Zhao, B. Bonello, L. Becerra, O. Boyko and R. Marchal, "Focusing of Rayleigh waves with gradient-index phononic crystals," Appl. Phys. Lett., vol. 108, (22), pp. 221905, 2016.

[3] X. F. Li, X. Ni, L. F. eng, M. H. Lu, C. He and Y. F. Chen, "Tunable Unidirectional Sound Propagation through a Sonic-Crystal-Based Acoustic Diode," Phys. Rev. Lett., vol. 106, (8), pp. 084301, 2011.

[4] P. Celli and S. Gonella, "Manipulating waves with LEGO (R) bricks: A versatile experimental platform for metamaterial architectures," Appl. Phys. Lett., vol. 107, (8), pp. 81901, 2015.

[5] P. H. Otsuka, K. Nanri, O. Matsuda, M. Tomoda, D. M. Profunser, I. A. Veres, S. Danworaphong, A. Khelif, S. Benchabane, V. Laude and O. B. Wright, "Broadband evolution of phononic-crystal-waveguide eigenstates in real- and k-spaces," Sci. Rep., vol. 3, pp. 3351, 2013. [6] T. Lin, C. Lin and J. Hsu, "Enhanced acousto-optic interaction in two-dimensional phoxonic crystals with a line defect," J. Appl. Phys., vol. 113, (5), pp. 53508, 2013.

[7] S. Benchabane, O. Gaiffe, R. Salut, G. Ulliac, V. Laude and K. Kokkonen, "Guidance of surface waves in a micron-scale phononic crystal line-defect waveguide," Appl. Phys. Lett., vol. 106, (8), pp. 81903, 2015.

[8] M. G. Baboly, A. Raza, J. Brady, C. M. Reinke, Z.C. Leseman and I. El-Kady, "Demonstration of acoustic waveguiding and tight bending in phononic crystals," Appl. Phys. Lett., vol. 109, (18), pp. 183504, 2016.

[9] Y. Pennec, B. Djafari-Rouhani, J. O. Vasseur, A. Khelif, and P. A. Deymier, "Tunable filtering and demultiplexing in phononic crystals with hollow cylinders," Phys. Rev. E, vol. 69, (4), pp. 046608, 2004.

[10] J. O. Vasseur, P. A. Deymier, B. Djafari-Rouhani, Y. Pennec, and A-C. Hladky-Hennion, "Absolute forbidden bands and waveguiding in two-dimensional phononic crystal plates," Phys. Rev. B, vol. 77, (8), pp.085415, 2008. 
[11] Y. Pennec, B. Djafari-Rouhani, H. Larabi, J. O. Vasseur, and A. C. Hladky-Hennion,

"Low-frequency gaps in a phononic crystal constituted of cylindrical dots deposited on a thin homogeneous plate," Phys. Rev. B, vol. 78, (10), pp.104105, 2008.

[12] T. -C. Wu, T.-T. Wu and J. Hsu, "Waveguiding and frequency selection of Lamb waves in a plate with a periodic stubbed surface," Phys. Rev. b, vol. 79, (10), pp. 104306, 2009.

[13] J. Sun and T. Wu, "Propagation of surface acoustic waves through sharply bent two-dimensional phononic crystal waveguides using a finite-difference time-domain method," Physical Review b, vol. 74, (17), pp. 174305, 2006.

[14] M. Oudich, B. Djafari-Rouhani, Y. Pennec, M. B. Assouar, and B. Bonello, "Negative effective mass density of acoustic metamaterial plate decorated with low frequency resonant pillars," J. Appl. Phys., vol. 116, (18), pp. 184504, 2014.

[15] M. Rupin, F. Lemoult, G. Lerosey, and P. Roux, "Experimental Demonstration of Ordered and Disordered Multiresonant Metamaterials for Lamb Waves," Phys. Rev. Lett., vol. 112, (23), pp. 234301, 2014.

[16] E. G. Williams, P. Roux, M. Rupin, and W. A. Kuperman, “Theory of multiresonant metamaterials for A0 Lamb waves", Phys. Rev. B, vol. 91, pp. 104307, 2015.

[17] W. Yuan, J. Zhao, B. Bonello, B. Djafari-Rouhani, X. Zhang, Y. Pan, and Z. Zhong, "Compact Waveguide and Guided Beam Pattern Based on the Whispering-Gallery Mode of a Hollow Pillar in a Phononic Crystal Plate”, Phys. Rev. Appl., vol. 10, pp. 034010, 2018.

[18] Y.-F. Wang, T.-T. Wang, and Y.-S. Wang, V. Laude, "Reconfigurable phononic-crystal circuits formed by coupled acoustoelastic resonators", Phys. Rev. Applied, vol. 8, pp. 014006, 2017.

[19] D. Zhang, J. Zhao, B. Bonello, F. Zhang, W. Yuan, Y. Pan and Z. Zhong, "Investigation of surface acoustic wave propagation in composite pillar based phononic crystals within both local resonance and Bragg scattering mechanism regimes," J. Phys. D: Appl. Phys., vol. 50, (43), pp. 435602,2017.

[20] D. Zhang, J. Zhao, B. Bonello, L. Li, J. Wei, P. Pan and Z. Zhong, “Air-coupled method to investigate the lowest-order antisymmetric Lamb mode in stubbed and air-drilled phononic plates.” AIP Adv., vol.6, (8), pp. 085021, 2016. 